\title{
A Modified General Diode Equation
}

\author{
Pragnan Chakravorty, Senior Member, IEEE
}

\begin{abstract}
The general diode equation or the non-ideal diode equation is the foundation of circuit models of active devices for the past several decades. Apart from the effect of p-n junction, this equation also accounts for the series bulk resistance of a diode. Despite a reasonable agreement of the equation with measured IV characteristics, it is shown here that the equation is incompatible with basic theories of circuits and systems. Therefore, a modification in the equation is proposed to remove this incompatibility. This modified equation leads to a compact model of a p-n junction diode that has an excellent agreement with the measured IV characteristics.
\end{abstract}

Index Terms - Diode circuit model, device modeling, compact model, semi-analytical model, ohmic transform, modified general diode equation, computer aided design

\section{INTRODUCTION}

$\mathrm{E}$ VER since the publication of Shockley's seminal research ${ }_{\text {on p-n junction diodes [1], general diode equation (GDE) }}$ has become the de-facto basis of device modeling and circuit simulations [2] enabled over computer simulation program with integrated circuit emphasis (SPICE). Unfortunately, the proposed solutions of this equation are mostly approximate [37]. Since an exact solution of the GDE has remained a challenge, most of the research on diode circuit modeling have focused on finding a solution of this equation [3-7]. So, research on verifying or improving the accuracy of the equation in prediction of practical IV characteristics have been very limited. Though the IV characteristics from GDE provide a fair estimate of the practical results, the need for higher accuracy has either led to the use of abstract mathematics [8] or use of multiple diodes [9 (i.e. multiple exponentiations [1011]) to model the behavior of a single diode. Consequently, device modeling has become more of an art than science as proclaimed in [12]. More appropriately, this art is mostly the use of empirical factors or relations that are qualitatively based on science and become quantitative only when matched with measured results. For example, the average velocity is a kind of empirical factor for a body moving with variable velocities; it becomes quantitative only after the total distance and total time of travel are measured. Such empirical factors are inevitable in many cases where the dynamics of science is obscure. The ideality factor $\eta$ is one such case which empirically predicts the combined effect of diffusion current

Manuscript received on Jul 02, 2021, final revision received on Aug 31, 2021 and accepted on Sep 23, 2021

Pragnan Chakravorty is currently with Clique for Applied Research in Electronic Technology, Bhilai, India. ( e-mail: pciitkgp@ieee.org) and recombination current on the IV characteristics of a diode [13]. Since it is presently impossible to determine the exact distribution of the total current in these two categories, the empirical nature of $\eta$ is inevitable. Therefore, such empirical factors or relations are an integral part of modern science and may not be deemed merely as art. However, many problems like those of radio propagation models [14] are so complex that their solutions are obtained purely using curve fitting, giving rise to only empirical formulations that are not even qualitatively connected with scientific understanding. Irrespective of whether such formulations are connected with science or not, it is important to ascertain that those are never in disagreement with established science. In the upcoming sections it is shown that GDE has disagreements with established theories of circuits and systems. Thereafter, a modified general diode equation (MGDE) is proposed which is fully compatible with the established theories and, unlike GDE, has an excellent agreement with measured results.

\section{LiMITATIONS OF THE GDE}

\section{A. In Context of Exact Solution}

If $i$ and $v$ are respective current and voltage of a device then an exact solution of its IV equation should be expressible in the form of $i=f(v)$ without any deliberation; therefore, as the exact solution claimed in [6] is not expressible in this form, it is not exact solution with respect to the sense mentioned here; [8] deliberately uses a Lambert W-function such that the basic structure of the GDE gets changed. Since, so far, the solution of GDE takes the form of $i=f(v, i)$, a solution of GDE still remains approximate in nature [3-7]. GDE does have an exact solution in terms of an unknown voltage, i.e. $v=f(i)$ where voltage across a diode may be obtained using an estimated current through it. Using this approach, named here as estimated GDE (E-GDE), IV curves of three different diodes are plotted and compared in Fig.1.

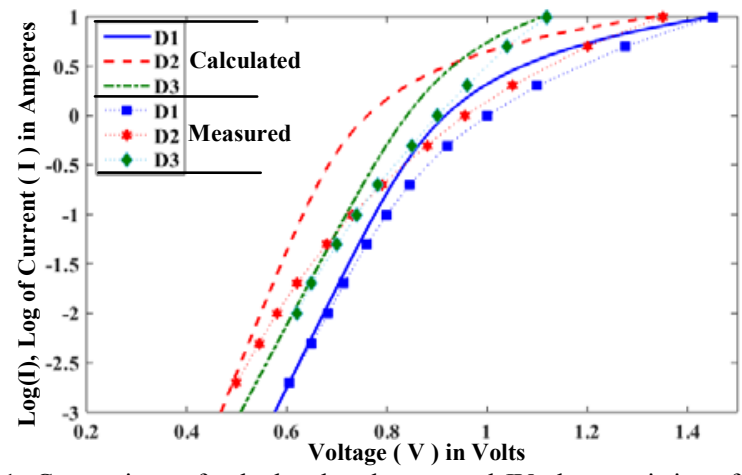

Fig.1. Comparison of calculated and measured IV characteristics of Diodes: D1 (IN5622), D2 (IN5420) and D3 (IN4001) using SPICE TABLE I. 
Measurements are obtained from published datasheets [15-17]. From the view point of rationality, what is known across a diode is the voltage and not the current. Therefore, the approach of an estimated output (i.e. current) and a calculated input (i.e. voltage) to solve the GDE is again an approximation. The GDE expressed in (1), irrespective of whether the solution of it is approximate or exact, is always iterative due to the presence of empirical factors like ideality factor $\eta$ and series bulk resistance $R_{b s}$. In (1), $I_{o}$ is the reverse saturation current and $V_{T}=k T / q$ is the thermal voltage related to physical parameters specified in standard references $[9,13]$. In (2), $V_{d}$ is the voltage across the diode, $V$ is the total voltage across the series circuit and $I$ is the total current through it. Usually, to closely match the solution of GDE with measurements, $I_{o}$ is also taken as an empirical factor in most of the SPICE parameter sheets; this practice is inappropriate because of the fact that $I_{o}$, in forward bias, is the measurable minimum reverse saturation current at a given temperature. Thus, the forward bias $I_{o}$ should at most be equal to the minimum $I_{o}$ obtained from measurements. Surprisingly, the values of these currents in SPICE sheets are over 100 times more than their measured values [18-19] indicating a disagreement with realistic conditions. Another unrealistic approximation in SPICE sheets is that of the values of $\eta$. These should be between 1 and 2 as recommended in the academic references [13]; however, the value of $\eta$ is more than 2 in many SPICE parameter sheets [18-19]. In the calculations adopted here, $I_{o}$ is directly taken from measurements (i.e. not empirically adjusted) and $\eta$ is estimated between 1 and 2 to obtain the closest match with measurements and to conform with rational estimates. These newly extracted SPICE parameters for the three different diodes are enlisted in TABLE I. The same set of diodes and their SPICE parameters is used in all the examples shown ahead.

$$
I=I_{o}\left(e^{\frac{V-I R_{b s}}{\eta V_{T}}}-1\right)
$$

where

$$
V=V_{d}+I R_{b s}
$$

TABLE I

EXTRACTED SPICE PARAMETERS FOR $25^{\circ}$ CELSIUS

\begin{tabular}{cccccc}
\hline \hline $\begin{array}{c}\text { Sr. } \\
\text { No. }\end{array}$ & $\begin{array}{c}\text { Name of } \\
\text { the Diode }\end{array}$ & Model No. & $\begin{array}{c}I_{o} \\
\text { (Amperes) }\end{array}$ & $\eta$ & $\begin{array}{c}R_{b s} \\
(\text { Ohms })\end{array}$ \\
\hline 1 & D1 & IN5622 & $1.25 \times 10^{-9}$ & 1.66 & $48.5 \times 10^{-3}$ \\
2 & D2 & IN5420 & $1.25 \times 10^{-9}$ & 1.35 & $55.0 \times 10^{-3}$ \\
3 & D3 & IN4001 & $14.0 \times 10^{-9}$ & 1.78 & $18.5 \times 10^{-3}$ \\
\hline
\end{tabular}

It is evident from Fig.1 that even the exact solution of GDE is not accurate enough to closely match the measured results.

\section{B. In Context of Circuit Theory}

The difference between the ideal diode equation (IDE), i.e. $I=I_{o}\left(e^{V / \eta V_{T}}-1\right)$, and the GDE must be clearly stated at this instant to appreciate the issues discussed ahead. While the IDE may be seen as a device equation, GDE is essentially a circuit equation formed by the series connection between the ideal diode and the bulk resistance. This is why Kirchhoff's voltage law (KVL) has been used to formulate the GDE. In other words, the general diode is a circuit rather than a single device. From Fig.1, it is amply clear that the solution of GDE provides a reasonable estimate of the actual forward IV characteristics of a diode for a large range of currents which the IDE cannot. Therefore, GDE leads to a large signal model whereas IDE is useful only for small signal approximations using $R_{d}=(d \mathrm{I} / d V)^{-1}$, a small signal dynamic resistance of the junction. Perhaps, this is the reason why validity of the GDE has never been questioned. However, a close examination of it in terms of circuit theory needs to be done in view of the fact that irrational estimates of the SPICE parameters bring more accuracy to the solution. It is obvious that the relation $V_{d}=V-$ $I R_{b s}$, used in the exponentiation of the GDE (1), is a KVL equation where current $I$ multiplied by $R_{b s}$ is the voltage drop across the bulk resistance as per ohm's law. So far, (1) seems to be compliant with circuit theory. An important aspect of a $\mathrm{KVL}$ equation is that the voltage drops in a circuit must be expressible in form of the ohm's law; however, the drop across the diode junction $V_{d}$ remains indeterminate within the GDE in context of the law. A deliberate attempt to write $V_{d}$ in terms of the law, i.e. $V_{d}=I R_{d}$, leads to (3) which, unfortunately, doesn't match with the measured results as shown in Fig.2. Apparently, a GDE must take the form of (3) to be compliant with KVL. Since it doesn't take this form, regardless of the signals being small or large, it is not compatible with circuit rules in general.

$$
I=\frac{V}{R_{d}+R_{b s}}
$$

It is important for the GDE to be compliant with circuit rules because in reality a diode cannot be operated in a circuit without the use of ohmic resistances. $R_{d}$ is the only defined resistance of the ideal diode and $V_{d}$ can't be expressed as $I R_{d}$ within the GDE, therefore, $R_{d}$ remains one of the bottlenecks in the formulation of a revised GDE that may be compliant with circuit theories and measured results.

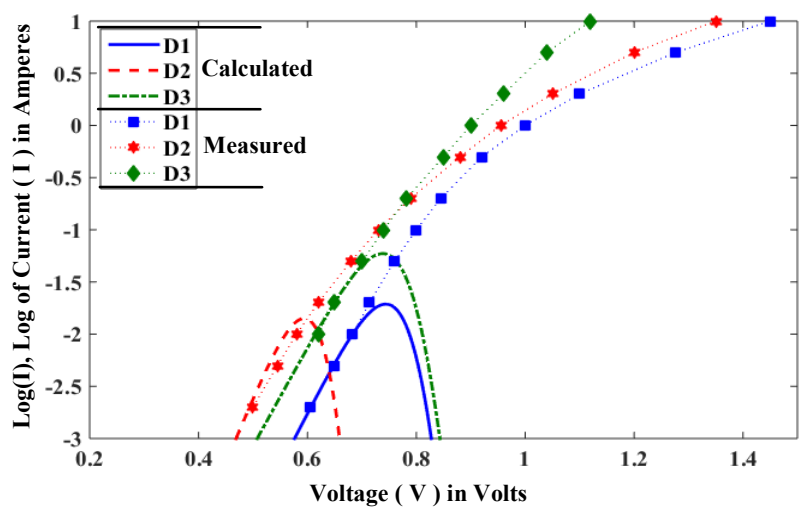

Fig. 2 The incompatibility of GDE with circuit theory.

\section{In Context of System Implementation}

It may be shown that if an equation is not expressible in the form of an exact solution, i.e. $i=f(v)$, a practical system of two or more devices may not be formed. Since the GDE 
doesn't offer an exact solution, as mentioned earlier, the practicality may be a problem here as well. This may be exemplified as follows. (1) expressed as (4) has $C=e^{V / \eta V_{T}}$. $C$ is the variable part of (4) that purely depicts the ideal diode. Thus, for a given value $C$, (4) must represent the behavior of the resistance $R_{b s}$ within the GDE as shown in Fig.3. A resistance is the simplest possible electrical system [20] where different voltages fed at different times are input signals [21] and the corresponding currents are the output signals. In that sense, current $I$ and the bulk resistance $R_{b s}$ must come under the purview of a defined signal through a defined system.

$$
\begin{gathered}
I=I_{o}\left(C e^{-I \frac{R_{b s}}{\eta V_{T}}}-1\right) \\
I_{o}\left(C e^{-I \frac{R_{b s}}{\eta V_{T}}}-1\right) \longrightarrow I
\end{gathered}
$$

Fig. 3 The incompatibility of GDE as a system equation.

As obvious from Fig.3, the depiction of the bulk resistance does not fit into the definition of any system. Because, for a system to exist, both input and output must exist. i.e. a single variable equation, like (4), has a single valued solution and can't represent a system. In terms of physics, a current (output) cannot exist through a resistor if a voltage (input) is not applied across it. As another example, if the ideal diode component is shorted (i.e. $C$ becomes infinite) then (4) must reduce to $I=V / R_{b s}$. Unfortunately, this does not happen. Had the GDE taken the format of (3), this disagreement with the reality would not have existed.

\section{THE MODIFIED GDE}

Now, it is clear that the GDE should not be acceptable in its present form, not just because it is approximate but also because it doesn't comply with the general rules of circuits and systems. So, the aim of this article is to propose a modified general diode equation (MGDE) that is fully compliant with prevalent theories, laws and rational estimations and the one that achieves a very good agreement with the measured results. It is commonly believed that circuit rules like KVL are applicable only to linear devices and not non-linear devices despite the fact that a KVL equation depicts conservation of energy that must be valid for non-linear devices as well. As mentioned earlier, this is because the defined resistances of these devices don't follow ohms law. For example, the ideal diode current (IDC) expressed in the form of the law as $I_{d}=V_{d}$ $/ R_{d}$, named as IDC with resistive transform (IDC-RT), does not agree with the actual IDE. This is shown in Fig.4. However, if the resistances of non-linear devices are so defined that they start following ohms law then the classification of non-ohmic devices may become unnecessary. To be precise, if $I_{d}$, expressible in the form of $I_{d}=V_{d} / R_{d o}$, is in agreement with the actual IDE, where $R_{d o}$ is a voltage dependent resistance, then a MGDE, fully compatible with the laws of circuits and systems, may easily be written. The ohm's law compliant $R_{d o}$ may be related to $R_{d}$ as $R_{d o}=\Theta R_{d}$ where $\Theta$ is defined as the ohmic transform factor. The exact expression of $\Theta$ may be readily obtained from (5). A compact form of it is given in (6) without any loss of accuracy for practical diodes. This form is obtained from (5) by neglecting only the subtracted portion of $I_{o}$. The total ohmic diode resistance $R_{t o}$ including the series bulk resistance $R_{b s}$ may now be expressed as (7). Fig.5 plots the general diode current with ohmic transform (GDC-OT), which is $I=V / R_{t o}$, and compares it with the measurements. $R_{d o}$ may be seen as the large signal equivalent of the small signal resistance $R_{d}$. It is important to note that (5) and other equations thereafter make a generalized interpretation of ohms law where the resistance of a device may not be a constant. Resistance is an empirically derived expression of proportionality that relates voltage and current across it. This expression, in most cases, is a constant because it is so observed. Therefore, when the same process of observation finds it variable, it should not be deemed as nonohmic. From another point of view, if a rheostat, i.e. a variable resistance, is believed to be compliant with all circuit theories including ohm's law then there is no reason to believe that the variable resistances of non linear devices are non-ohmic. Practically, resistances like $R_{d}$ are electrically controlled rheostats.

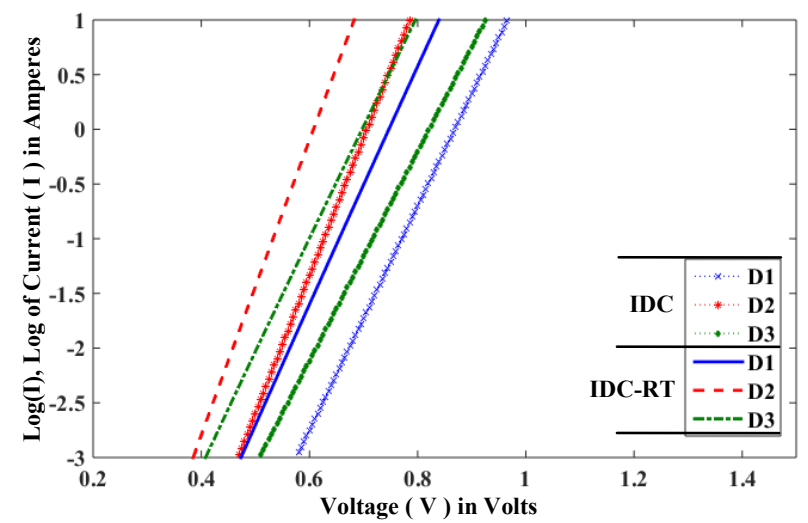

Fig. 4 Ideal diode current (IDC) and with resistive transform (IDC-RT).

$$
\begin{aligned}
\frac{V}{\Theta R_{d}} & =I_{o}\left(e^{\frac{V}{\eta V_{T}}}-1\right) \\
\Theta & \cong \frac{V}{\eta V_{T}} \\
R_{t o} & =\Theta\left(R_{d}+R_{b s}\right)
\end{aligned}
$$

The mismatch in the calculated and measured results of Fig.5 is attributed to the use of constant bulk resistance. The bulk resistance $R_{b s}$ should also be a variable like $R_{d}[12,22]$ because any resistance that is forced to process a non linear current through itself cannot remain fixed. This may be termed as an induced variability in an otherwise constant resistance. Since the non-linear current, generated by the junction, is forced through $R_{b s}$ connected in series with it, $R_{b s}$ must 
become a variable that decreases with increasing voltage so as to match the outcome of the actual IV characteristics.

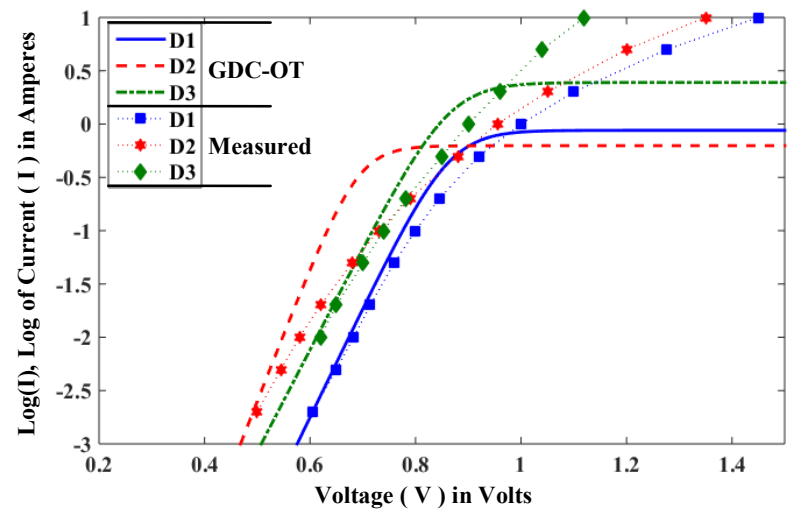

Fig. 5 The results after ohmic transform and addition of $R_{b s}$.

Consequently, a general scaling factor $S_{G}$ is formulated in (8) which when divided with the bulk resistance, provides the desired result. The $S_{G}$ may be composed of a static part $S_{s}$ and a dynamic part dependent on the voltage. the factor $m$ in (8) may be called the scaling order. Since, no resistance should reduce down to zero, a damping factor $\sigma$ is introduced to retard this process so that the final value of the bulk resistance settles near its initial static value. The factor $I_{o} R_{b s}$ may be interpreted as the minimum voltage required to establish a non zero current through the diode. Practically, this non zero current is the leakage current $I_{o}$. The minimum voltage also ensures that that the variable bulk resistance is not mathematically infinite at zero forward bias voltage.

$$
S_{G}=S_{s}\left(I_{o} R_{b s}+V\right)_{N}^{m /\left(1+\sigma V_{N}\right)}
$$

$S_{G}$ may also be seen as a current amplification factor of the bulk because it increases the current through the circuit by reducing the effective resistance. It may be noted that all the newly introduced empirical factors, $m, S_{s}$ and $\sigma$ in $S_{G}$ are unitless quantities. The other entities used in it are made unitless by the process of normalization against their unit quantities as indicated by their subscript $N$. Now we may write the MGDE in its compact form as in (9)

$$
I=\frac{V}{\Theta\left(R_{d}+\frac{R_{b s}}{S_{G}}\right)}
$$

TABLE II shows the three new empirical factors, for the present set of diodes, which may be adopted as ancillary SPICE parameters in standard modeling practices.

TABLE II

EXTRACTED ANCILLARY SPICE PARAMETERS FOR $25^{\circ}$ CELSIUS

\begin{tabular}{cccccc}
\hline \hline $\begin{array}{r}\text { Sr. } \\
\text { No. }\end{array}$ & $\begin{array}{c}\text { Name of } \\
\text { the Diode }\end{array}$ & Model No. & $S_{s}$ & $m$ & $\sigma$ \\
\hline 1 & D1 & IN5622 & 1.176 & 6.3 & 0.01 \\
2 & D2 & IN5420 & 2.041 & 8.8 & 0.20 \\
3 & D3 & IN4001 & 1.428 & 9.1 & 0.01 \\
\hline
\end{tabular}

Similar to the case of E-GDE, the empirical parameters of MGDE may be extracted by means of curve fitting (9) to the measured data [15-17]. Here, the values of $\eta, I_{o}$ and $R_{b s}$ are taken from TABLE I and the ancillary parameters of TABLE II are extracted over manual curve fitting trials. The extracted parameters from these trials are usually made accurate using a regression method like least squares [23]. Automation and higher accuracy of the extractions may be attained using computer aided optimization algorithms [24]. The solution of (9) has an excellent agreement with measured results as shown in Fig.6.

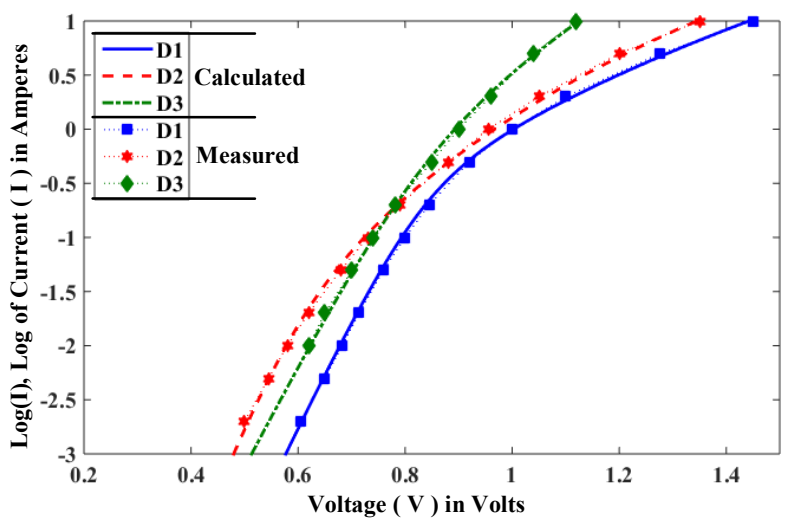

Fig. 6 The IV characteristics from MGDE compared to those of measurements

\section{COMPARISON OF PERFoRMANCES}

Observations and conclusions made so far enable the comparison of the performances of different diode equations, viz. IDE, GDE, E-GDE and MGDE, as given in TABLE III.

TABLE III

COMPARISON TABLE

\begin{tabular}{cccccc}
\hline \hline $\begin{array}{c}\text { Sr. } \\
\text { No. }\end{array}$ & Properties & IDE. & GDE & E-GDE & MGDE \\
\hline 1 & Solution Type & Exact & Approximate & Exact & Exact \\
2 & $\begin{array}{c}\text { Iterative } \\
\text { Parameters }\end{array}$ & $\eta$ & $I, \eta, I_{o}, R_{b s}$ & $\eta, I_{o}, R_{b s}$ & $\eta, m, S_{s}, \sigma$ \\
3 & $\begin{array}{c}\text { Computational } \\
\text { Effort }\end{array}$ & Low & High & Medium & Medium \\
& Input $(V)$ & Known & Known & Calculated & Known \\
4 & Output $(I)$ & Calculated & Calculated & Estimated & Calculated \\
5 & $\begin{array}{c}\text { Theoretical } \\
\text { Correctness }\end{array}$ & Yes & No & No & Yes \\
7 & $\begin{array}{c}\text { Parametric } \\
\text { Values }\end{array}$ & Realistic & Unrealistic & Unrealistic & Realistic \\
8 & Agreement & & & & \\
& with & Poor & Fair & Fair & Excellent \\
& Measurements & & & & \\
\hline
\end{tabular}

All the diode equations contain one or more empirical parameters and, as a result, all of them require curve fitting. Consequently, none of them are purely analytical. Their primary dependence on temperature is governed by the thermal voltage $V_{T}=k T / q$. Dependence on materials is brought in by the factors like conductivity, resistivity, mobility and so on. These material dependent factors also depend on temperature [25]. $I_{o}$ and $R_{b s}$ are not iterative in the MGDE. $I_{o}$ is taken from measurements and $R_{b s}$ may be calculated using 
physical dimensions of the bulk and its resistivity. It is now evident that the MGDE has clear advantages over the other diode equations. All the advantages of this new equation could be achieved at the cost of one iterative parameter more than that of the DC SPICE model based on E-GDE. Therefore, the computational effort in extracting the parameters of MGDE is insignificantly more than that of E-GDE approach. Without the E-GDE approach, solution of the GDE is approximate and the calculation of the current $I$ itself becomes iterative. So, the solution of the GDE as such takes the highest computational effort. Conclusively, the MGDE provides a compact model for computer aided designs of practical diodes in a much better way than ever before.

\section{CONCLUSION}

A generalized view of the ohm's law leads to the interpretation of resistance as an electrical ratio of voltage upon current which may not always be a positive constant. This interpretation puts the category of non-ohmic resistances in disuse. i.e. non-linear resistances such as $R_{d}$ or $R_{b s}$ of general diodes or negative resistances of devices like tunnel diodes may all be now assumed as ohmic resistances. A modified general diode equation, MGDE, is proposed whereby a non ideal diode may be modeled as a combination of the ideal diode resistance $R_{d}$ and the series bulk resistance $R_{b s}$. To make the MGDE compliant with the laws of circuits and systems, it uses an analytical transform, $\Theta$, named as ohmic transform. The transform enables the diode resistances to behave like normal resistors compliant with ohm's law. Conforming with realistic conjectures [22], the series bulk resistance is made variable like the existing ideal diode resistance. This results in a MGDE that is apparently the simplest yet the most accurate in the fundamental circuit modeling of semiconductor devices.

\section{REFERENCES}

[1] W. Shockley, "The theory of p-n junctions in semiconductors and p-n junction transistors," The Bell System Technical Journal, vol. 28, no. 3, pp. 435-489, July 1949.

[2] L. W Nagel and D. O Pederson, "Simulation Program with Integrated Circuit Emphasis (SPICE)," University of California, Berkeley Technical Report No. UCB/ERL M382, April 1973.

[3] T. A. Fjeldly, B. -. Moon and M. Shur, "Approximate analytical solution of generalized diode equation," IEEE Transactions on Electron Devices, vol. 38, no. 8, pp. 1976-1977, Aug. 1991.

[4] J. M. Pimbley, T. A. Fjeldly, B. Moon and M. Shur, "Iterative solutions of the generalized diode equation," IEEE Transactions on Electron Devices, vol. 39, no. 5, pp. 1268-1269, May 1992

[5] Jin He et al., "A new analytic approximation to general diode equation", Solid-State Electronics, vol. 50, no. 7-8, , pp.1371-1374, July-August 2006

[6] J. Le Bihan, "Accurate analytical approximation for normalized diode characteristic", International Journal Of Circuit Theory And Applications, vol. 29, no. 4, , pp. 397-401, July 2001

[7] A. Ortiz-Conde and F. J Garcia Sanchez, "Approximate analytical expression for equation of ideal diode with series and shunt resistances". Electronics Letters, vol. 28, no. 21, pp. 1964 -1965, October 1992

[8] T. C. Banwell and A. Jayakumar, "Exact analytical solution for current flow through diode with series resistance," Electronics Letters, vol. 36, no. 4, pp. 291-292, 17 Feb. 2000.

[9] C.G. Fonstad, Microelectronic Devices and Circuits, MA: Copyright (C) Fonstad, 2006, pp.157
[10] B.V. Zeghbroeck, Principles of Semiconductor Devices, CO: Copyright (C) Zeghbroeck, 2011, ch.4.4.4

[11] C. Cane et al., "An easy technique for determining diffusion and generation-recombination components of the current of pn junctions for better modelling," ICMTS 93 Proceedings of the 1993 Int. Conference on Microelectronic Test Structures, Sitges,1993, pp. 167-170

[12] L.O Chua, "Device Modeling Via Basic Nonlinear Circuit Elements," IEEE Transactions on Circuits and Systems, vol. cas-27, no. 11, pp. 1014-1044, Nov. 1980

[13] K. Kano, Semiconductor Devices, Prentice Hall, New Jersey, 1998, ch.7

[14] T. K. Sarkar et al., "A survey of various propagation models for mobile communication," IEEE Antennas and Propagation Magazine, vol. 45, no. 3, pp. 51-82, Jun. 2003

[15] 1N5614-1N5622 Data Sheet, Microsemi, AZ: C2007 1-15-2007, 2007

[16] 1N5415-1N5420 Data Sheet, Microsemi Corp, CA

[17] 1N4001-1N4007 Data Sheet, ON Semiconductor, AZ: C2012

[18] 1N5622 SPICE Model, Voltage Multipliers Inc., CA

[19] 1N5420 SPICE Model, Voltage Multipliers Inc., CA

[20] S. S. Haykin and B. V. Veen, Signals \& Systems, Hoboken, NJ: Wiley, 2003

[21] P. Chakravorty, "What Is a Signal? [Lecture Notes]," IEEE Signal Processing Magazine, vol. 35, no. 5, pp. 175-177, Sept. 2018

[22] A. Barna and D. Horelick, "A simple diode model including conductivity modulation," IEEE Transactions on Circuit Theory, vol. 18, no. 2, pp. 233-240, Mar. 1971.

[23] W.Y Yang et al., Applied Numerical Methods Using MATLAB, JohnWiley, New Jersey, 2005, ch.3

[24] P. Chakravorty and D. Mandal, "Role of Boundary Dynamics in Improving Efficiency of Particle Swarm Optimization on Antenna Problems," 2015 IEEE Symposium Series on Computational Intelligence, Cape Town, 2015, pp. 1157-1163

[25] N.D. Arora et al., "Electron and hole mobilities in silicon as a function of concentration and temperature", IEEE Transactions on Electron Devices, vol. 29, no.2, pp 292-295, Feb. 1982. 